\title{
Open water scuba diving accidents at Leicester: five years' experience
}

\author{
A J Hart, S A White, P J Conboy, G Bodiwala, D Quinton
}

\begin{abstract}
Objectives-The aim of this study was to determine the incidence, type, outcome, and possible risk factors of diving accidents in each year of a five year period presenting from one dive centre to a large teaching hospital accident and emergency (A\&E) department.
\end{abstract}

Methods-All patients included in this study presented to the $A \& E$ department at a local teaching hospital in close proximity to the largest inland diving centre in the UK. Our main outcome measures were: presenting symptoms, administration of recompression treatment, mortality, and postmortem examination report where applicable.

Results-Overall, 25 patients experienced a serious open water diving accident at the centre between 1992 and 1996 inclusive. The percentage of survivors $(n=18)$ with symptoms of decompression sickness receiving recompression treatment was $52 \%$. All surviving patients received medical treatment for at least 24 hours before discharge. The median depth of diving accidents was 24 metres (m) (range 7-36 $\mathrm{m})$. During the study period, 1992-96, the number of accidents increased from one to 10 and the incidence of diving accidents increased from four per 100000 to 15.4 per 100000 . Over the same time period the number of deaths increased threefold. Conclusions-The aetiology of the increase in the incidence of accidents is multifactorial. Important risk factors were thought to be: rapid ascent (in $48 \%$ of patients), cold water, poor visibility, the number of dives per diver, and the experience of the diver. It is concluded that there needs to be an increased awareness of the management of diving injuries in an $A \& E$ department in close proximity to an inland diving centre.

(F Accid Emerg Med 1999;16:198-200)

Keywords: scuba diving; decompression sickness; barotrauma; nitrogen narcosis

Open water scuba diving is becoming an increasingly popular recreational activity. ${ }^{1}$ The nature of the sport includes rapidly changing ambient pressures, resulting in physiological stresses that can produce dangerous sequelae. Decompression sickness is reported to occur in approximately one in 15000 dives. $^{1}$

In general, serious accidents at inland diving centres are primarily dealt with by the nearest accident and emergency (A\&E) department, which in some cases involves dispatching a medically led flying squad to the scene of the accident. The largest inland diving centre in England is near to large centres of population and a teaching hospital, and as a flooded quarry is unusually deep. The diving centre has its own rescue boat led by a crew trained in basic life support; in addition there are on site facilities for immediate recompression if necessary. The temperature remains constant between $3-4^{\circ} \mathrm{C}$ at a maximum depth of 35 metres (m). The aim of this study was to determine the incidence, nature, outcome, and possible risk factors of diving accidents in each year of a five year period presenting from one dive centre to a large teaching hospital $A \& E$ department.

\section{Methods}

Details of all patients experiencing an open water scuba diving accident from 1992-96 inclusive were retrieved from the $A \& E$ audit database of a large teaching hospital. These incidents were confirmed by records kept at the regional dive centre. A diving accident was defined as symptoms within one day of diving, requiring medical treatment or observation for at least a 24 hour period. Diving accidents after dives from other diving centres were excluded. Where necessary details of hospital inpatient treatment were also documented. The number of divers in each year was obtained directly from the regional dive centre database. The figures for 1992 are approximate because the dive centres database was not computerised until 1993. The dive ascent profile was obtained from either the diver's own dive computer or buddy diver, details of which were recorded in the $A \& E$ notes. The postmortem examination reports were obtained from the coroner and local consultant pathologists.

A recent consensus has emerged for the classification of diving injuries, ${ }^{2}$ which we applied to our study. The injuries were categorised by description of the symptoms experienced and the signs found on examination: pain, neurological, cutaneous, audiovestibular, pulmonary, and constitutional. Pain experienced was usually dull, affecting large joints but occasionally girdle and loin pain. Neurological manifestations included almost any pattern of peripheral and central dysfunction. Audiovestibular manifestations included vertigo, nystagmus, vomiting, nausea, and tinnitus. Pulmonary manifestations included chest pain ${ }_{2}$ cough, dyspnoea, haemoptysis, and cyanosis. Cutaneous manifestations were those usually induced by barotrauma. Constitutional manifestations were less specific and included anorexia, malaise, fatigue, and headache. 
Table 1 Summary of study results

\begin{tabular}{llllll}
\hline Year & $\begin{array}{l}\text { No of } \\
\text { divers }\end{array}$ & No of accidents & $\begin{array}{l}\text { Incidence of accidents } \\
\text { (per 100 000 divers) }\end{array}$ & No of deaths & $\begin{array}{l}\text { Incidence of deaths } \\
\text { (per 100 000 divers) }\end{array}$ \\
\hline 1992 & 25118 & 1 & 4 & 1 & 4.0 \\
1993 & 41087 & 2 & 4.9 & 0 & 0 \\
1994 & 51125 & 4 & 7.8 & 1 & 2.0 \\
1995 & 56071 & 8 & 14.3 & 2 & 3.6 \\
1996 & 65100 & 10 & 15.4 & 3 & 4.6 \\
Total & 238501 & 25 & 10.5 & 7 & 2.9 \\
\hline
\end{tabular}

\section{Results}

The results are summarised in table 1 . Over the study period there were 25 diving accidents, seven of which resulted in death at the scene. Overall $65 \%$ were male, $35 \%$ were female, and the median age was 34 years (range 19-50 years). The median depth at which accidents occurred was $24 \mathrm{~m}$ (range 7-36 m). A significant number of accidents involved rapid dive ascent profiles ( $48 \%$ ) compared with $24 \%$ with a normal ascent profile $(28 \%$ were unknown). The overall rate of recompression for the survivors was $52 \%$. Recompression took place at the regional dive centre (which has non-resident medical cover) and in severe cases multiple recompressions were also undertaken at more distant ( $>120$ miles) medical hyperbaric units after inpatient hospital assessment at Leicester. The number of divers visiting the centre increased from 25118 to 65100 over the study period (fig 1). The number of accidents and deaths also increased (fig 2). There was an increase in the incidence of accidents but no significant increase in the incidence of deaths (fig 3).

The most common presenting symptoms experienced by the survivors $(n=18)$ were neurological $(52 \%)$, ranging from complete paraplegia in extreme cases to transient memory loss in mild cases. Pulmonary symptoms $(23 \%)$ ranged from severe pulmonary oedema to minor haemoptysis. Audiovestibular symptoms $(7 \%)$ ranged from ruptured tympanic membrane to mild dizziness. Pain $(7 \%)$ ranged from severe abdominal pains to milder joint pains. Constitutional symptoms (7\%) included headaches and vomiting. In the seven

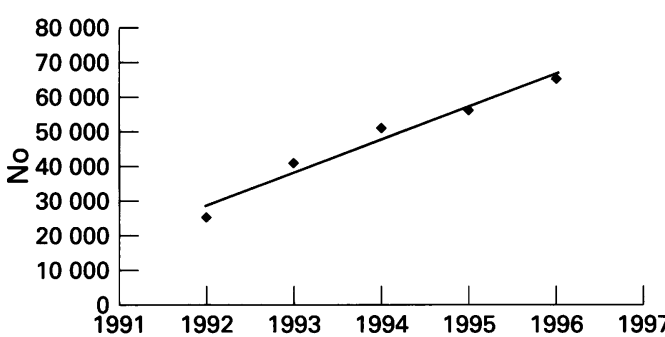

Figure 1 Number of divers attending the dive centre from 1992-96.

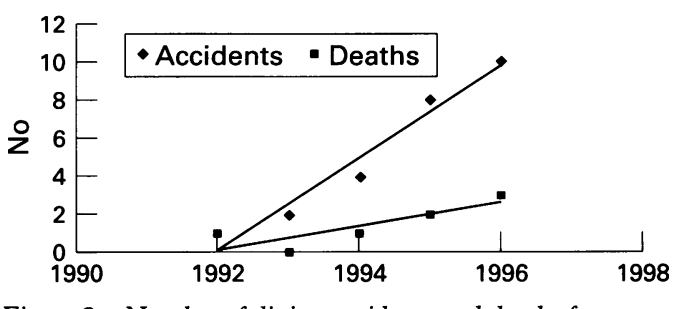

Figure 2 Number of diving accidents and deaths from 1992-96.

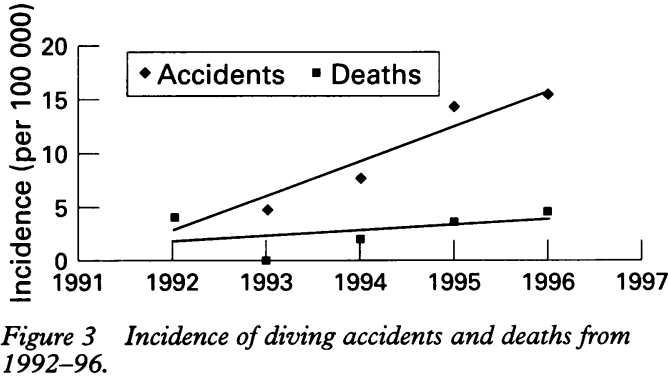

patients who died a precise history could not be obtained. Of these four died before surfacing and three had a respiratory or cardiac arrest very soon after surfacing.

Postmortem examinations were performed in all non-survivors, revealing haemorrhagic pulmonary oedema consistent with drowning. Finally, one of these patients also showed signs of acute metabolic liver failure of an unknown cause.

\section{Discussion}

The pathophysiology of scuba diving accidents is related to the rapidly changing ambient pressures. There are three potential mechanisms involved, in otherwise healthy individuals. Firstly there is barotrauma, defined as tissue injury resulting from failure of gas filled spaces (lungs, middle ear, and sinuses) to equalise their pressure with the ambient pressure. Pulmonary barotrauma is the most severe form and is potentially life threatening when arterial gas emboli reach the systemic circulation ${ }^{3}$ or when pneumothorax occurs. The main risk factor for pulmonary barotrauma is rapid ascent but this may occur in healthy divers after a normal ascent. ${ }^{3}$ Secondly, there is nitrogen narcosis where the raised partial pressure of nitrogen in the central nervous system induces a clinical picture similar to alcohol intoxication. And lastly there is decompression sickness, where the increased dissolved nitrogen (due to increased partial pressure at depth below the surface) is released as a gas at the lower ambient pressures on the surface resulting in occlusion of arteries, veins, and lymphatics of any organ. The risk factors for developing decompression sickness are those that enhance the accumulation of inert gases in tissues and include age, female sex, obesity, increased arterial carbon dioxide, low water temperature, poor physical fitness, and level of physical activity during the dive. ${ }^{4}$ Dives repeated in series are a major risk factor as after one dive considerable nitrogen may remain in the tissues. $^{5}$

There has been considerable recent debate on how to categorise the type of diving accident. For example neurological symptoms may arise from either decompression sickness or arterial gas embolus (after pulmonary barotrauma and gas dissolving into the pulmonary veins). The wide range of symptoms has led to the suggestion that specialist advice should be sought at a low threshold from the 24 hour service at the Institute of Naval Medicine ${ }^{6}$ (see address at end of paper). Our experience has taught us that this is the single most helpful 
approach to the management of diving injuries together with the important first aid treatments of $100 \%$ oxygen and rehydration. ${ }^{6}$ In our cohort the percentage of divers with symptoms of decompression sickness who received recompression $(52 \%)$ is considerably lower than that quoted by Wilmshurst et al ${ }^{1}$ perhaps suggesting a lower threshold for referral to specialist centres by junior A\&E staff. Melamed et al have emphasised the need for general practitioners and $A \& E$ staff to be acquainted with the basics of diving medicine because of the increasing popularity of the sport in the United States, so as to improve the quality of medical care for those suffering from diving related injuries. $^{\text {? }}$

To fully exclude the lack of medical fitness as a risk factor for diving accidents is difficult in a retrospective study. The evidence in our study suggests that medical disease was important in only one diver. Firstly, diving at the centre is only allowed with a British sub-aqua club or Professional Association of Dive Instructors (PADI) qualification, both of which involve a very detailed medical questionnaire with subsequent medical examination if indicated. Secondly, there was no relevant past medical history (for example asthma, diabetes) from the notes of the divers. Lastly, six of the seven postmortem examinations concluded that death was due to drowning in the absence of any other medical problem. Medical disease was thought to be significant in one women diver, a qualified dive instructor with a body mass index of 27 whose postmortem examination revealed her to have a fatty liver, raising the possibility of a metabolic liver disease contributing to her death. A fatty liver is also associated with alcohol intake, which may indicate that the diver was less likely to abstain from alcohol in the 12 hours before her dive, a common problem with amateur divers. Indeed, one of the accidents in this study involved a 30 year old man known to have drunk more than 10 pints of beer the night before his Sunday morning dive that resulted in paraplegia.

The pathogenesis in the remaining accidents involves one or a combination of barotrauma, decompression sickness, or nitrogen narcosis. The main risk factor for barotrauma and decompression sickness that we can identify in our cohort is rapid ascent, which is potentially preventable. Other risk factors for decompression sickness in our cohort include low water temperature $\left(4^{\circ} \mathrm{C}\right.$ at $30 \mathrm{~m}$ depth $)$, poor visibility $(1 \mathrm{~m}$ at $30 \mathrm{~m})$, and multiple dives in series. Another likely factor is the high proportion of inexperienced divers because the dive centre is very popular with those taking the course for PADI, which can be completed in only five days. After passing this course divers can dive without supervision, but it is not advised.

An important individual risk factor is the number of dives undertaken by each diver. It is difficult to accurately assess this retrospectively but the number of dives have increased dramatically over the study period. One can therefore assume that divers are performing an increasing number of dives and that more individuals are participating in open water diving; this latter trend has been noted previously in the UK. ${ }^{1}$ As already discussed, the experience of the diver is another important factor and with the increasing popularity of the sport one can speculate that an increasing number of inexperienced divers are perhaps participating in diving without appreciating the considerable risks involved.

The actual numbers of accidents may be higher than we have reported if divers presented to other $A \& E$ departments remote from the centre studied after their journey home. Occasionally symptoms related to decompression sickness may be precipitated by air travel resulting in presentation to hospitals even further away and close to airports.

In conclusion, this study demonstrates a trend of increasing accidents and deaths from recreational open water scuba diving in young individuals. The risk factors discussed are multifactorial and include inexperience, rapid ascent, the number of dives per diver, and cold water with poor visibility. Some of these risk factors are preventable but all should feature prominently in the education of divers. In order to prevent the rise in diving related injuries there needs to be an increased awareness of the potential dangers of dysbaric syndromes among divers and of their management among doctors.

Emergency Services, Institute of Naval Medicine, Alverstoke, Gosport PO12 2DL; tel: 01705768051 (24 hours).

Conflict of interest: none.

Funding: none.

1 Wilmshurst P, Allen C, Parish T. Incidence of decompression illness in amateur scuba divers. Health Trends 1994;26:116-18.

2 Smith DJ, Francis TJR, Pethybridge RJ, et al. Concordance: a problem with the current classification of diving a problem with the current classification of diving
disorders. Undersea Biomedical Research 1992;19(suppl):40.

3 Dutka AJ. A review of the pathophysiology and potential Dutka AJ. A review of the pathophysiology and potential
application of experimental therapies for cerebral ischaeapplication of experimental therapies for cerebral ischaemia to the treatment of cerebral arterial gas
Undersea Biomedical Research 1985;12:403-21.

4 Hills BA. Decompression sickness. Vol 1. The biophysical basis of prevention and treatment. Chichester: John Wiley, 1977.

5 Department of the Navy. US Navy diving manual. Washington, DC: Department of the Navy, 1985 (NAVSEA 0994LP-001-9010).

6 Sykes JJW. Medical aspects of scuba diving. BMF 1994;308: 1483-7.

7 Melamed Y, Shupak A, Bitterman H. Medical problems associated with underwater diving. $N$ Engl $\mathcal{F}$ Med 1992;326: 